\title{
Balancing the Promises and Perils of Artificial Intelligence and Machine Learning: Disruption, Adoption, Dehumanization, Governance, Risk and Compliance
}

\author{
Valeria Sadovykh \\ University of Auckland \\ valeriasadovykh@gmail.com
}

\author{
Kevin Craig \\ University of Auburn \\ kac0117@auburn.edu
}

\author{
David Sundaram \\ University of Auckland \\ d.sundaram@auckland.ac.nz
}

In the last decade, Artificial Intelligence (AI) and Machine Learning (ML) have developed from peripheral technologies to dominant drivers of innovation. They are routinely used to recognize images; parse speech; respond to questions; make decisions; and replace humans.

Given that AI and ML tools are becoming a part of our everyday lives, it is critical that researchers and practitioners understand their state of art, adoption and influence. Improperly deployed AI and ML tools can violate privacy, threaten safety, and take questionable decisions that can affect individuals, organizations and ultimately society.

This mini track focus is on the promises and perils of AI and ML with a particular emphasize on (a) adoption, (b) disruption, (c) potential dehumanization, and (c) governance, risk and compliance mechanisms required to protect and enhance human wellbeing. We welcomed wideranging papers with qualitative and quantitative orientations; with theoretical and practical contributions; from personal, organizational and societal perspectives.

This mini track has been running for more than 3 years and continues to attract interesting and futuristic papers. This year we had a number of submissions with a diverse range of topics. The three selected papers investigate key issues that exist in the research area.

The first paper, Tracing the Legitimacy of Artificial Intelligence - A Media Analysis, 1980-2020? Artificial Intelligence (AI) has received ambivalent evaluations, ranging from AI as a great opportunity and solution to crucial problems of our time to AI as a threat to humanity. For AI technologies to diffuse, they need to gain legitimacy. The study traced the legitimacy of AI in society from 1980 to 2020 . The study reviewed 2,543 newspaper articles from The New York Times as a reflection of societal discourse over the legitimacy of AI. Using computer-assisted content analysis, the authors found a sharp increase in media coverage around the mid-2010s. The findings showed that the language used in the articles to be predominantly positive and to show little changes over time.

The second paper, Perceived Social Media Bias, Social Identity Threat, and Conspiracy Theory Ideation During the COVID-19 Pandemic. The paper has discussed how social media organizations have an obligation to filter and sometimes exclude content, often based on machine learning algorithms. This has resulted in perceptions of bias in social media. When individuals perceive that a social media system is designed to exclude their point of view, they may experience a loss of self-worth, based 
on their excluded point of view. As a result, they may resist and avoid the technology that seems biased against them to prevent further loss of self-worth. They might also believe in conspiracies about why social media is marginalizing their point of view and find new self-worth as a conspiracy theorist. Data from 225 individuals who are interested in the risks associated with vaccines indicate that Perceived Bias presents a Social Identity Threat, which, in turn, is associated with Resistance to IT and Conspiracy Theorist Ideation. 